\title{
Ultrasound Guidance for Renal Tract Access and Dilation Reduces Radiation Exposure during Percutaneous Nephrolithotomy
}

\author{
Thomas Chi, ${ }^{1}$ Selma Masic, ${ }^{1}$ Jianxing $\mathrm{Li}^{2},{ }^{2}$ and Manint Usawachintachit ${ }^{1,3}$ \\ ${ }^{1}$ Department of Urology, University of California, San Francisco, 400 Parnassus Avenue, Suite A610, P.O. Box 0330, \\ San Francisco, CA 94143, USA \\ ${ }^{2}$ Department of Urology, Tsinghua Changgung Hospital, Beijing 100034, China \\ ${ }^{3}$ Division of Urology, Department of Surgery, Faculty of Medicine, Chulalongkorn University, King Chulalongkorn Memorial Hospital, \\ Rama IV Road, Patumwan, Bangkok 10330, Thailand
}

Correspondence should be addressed to Thomas Chi; tom.chi@ucsf.edu

Received 29 October 2015; Revised 10 February 2016; Accepted 14 February 2016

Academic Editor: Mohammad H. Ather

Copyright (C) 2016 Thomas Chi et al. This is an open access article distributed under the Creative Commons Attribution License, which permits unrestricted use, distribution, and reproduction in any medium, provided the original work is properly cited.

Purposes. To present our series of 38 prone percutaneous nephrolithotomy procedures performed with renal access and tract dilation purely under ultrasound guidance and describe the benefits and challenges accompanying this approach. Methods. Thirty-eight consecutive patients presenting for percutaneous nephrolithotomy for renal stone removal were included in this prospective cohort study. Ultrasonographic imaging in the prone position was used to obtain percutaneous renal access and guide tract dilation. Fluoroscopic screening was used only for nephrostomy tube placement. Preoperative, intraoperative, and postoperative procedural and patient data were collected for analysis. Results. Mean age of patients was $52.7 \pm 17.2$ years. Forty-five percent of patients were male with mean BMI of $26.1 \pm 7.3$ and mean stone size of $27.2 \pm 17.6$ millimeters. Renal puncture was performed successfully with ultrasonographic guidance in all cases with mean puncture time of $135.4 \pm 132.5$ seconds. Mean dilation time was $11.5 \pm 3.8$ min and mean stone fragmentation time was $37.5 \pm 29.0 \mathrm{~min}$. Mean total operative time was $129.3 \pm 41.1$. No patients experienced any significant immediate postoperative complication. All patients were rendered stone-free and no additional secondary procedures were required. Conclusions. Ultrasound guidance for renal access and tract dilation in prone percutaneous nephrolithotomy is a feasible and effective technique. It can be performed safely with significantly reduced fluoroscopic radiation exposure to the patient, surgeon, and intraoperative personnel.

\section{Introduction}

Percutaneous nephrolithotomy (PCNL) is a procedure commonly performed by urologists worldwide. Since its first introduction by Fernström and Johansson in 1976, it has become the mainstay surgical treatment for renal stones larger than 2 centimeters and those refractory to shockwave therapy [1]. Nevertheless, one concern related to this procedure is the possible long-term effects of ionizing radiation exposure sustained by the surgeon, medical personnel, and patient during the operation which is usually guided by fluoroscopic imaging $[2,3]$. Numerous studies have shown that occupational exposure dose during fluoroscopic procedures can be kept within safe limits with routine use of protective aprons and thyroid shields, but some degree of radiation exposure to intraoperative personnel can still be detected [4-6]. One main factor influencing exposure is fluoroscopic screening time, which is significantly affected by total stone burden and the need for multiple accesses [7].

The utilization of ultrasound (US) can obviate the need for ionizing radiation exposure intraoperatively and provide a reliable method for the localization of renal stones. It can help surgeons create an appropriate access into the collecting system via a posterior calyx, guide tract dilation, and even confirm stone clearance after the procedure is completed. In addition, it can be an ideal imaging modality for special 


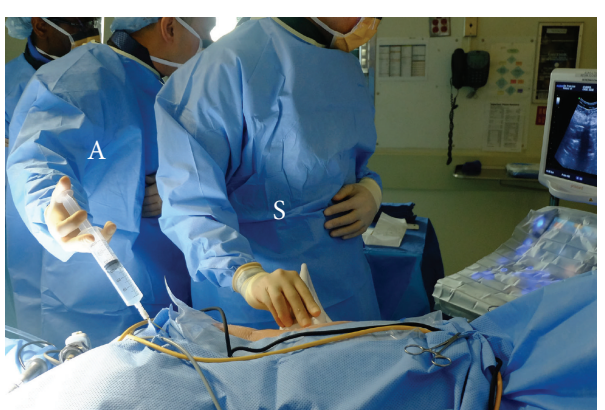

(a)

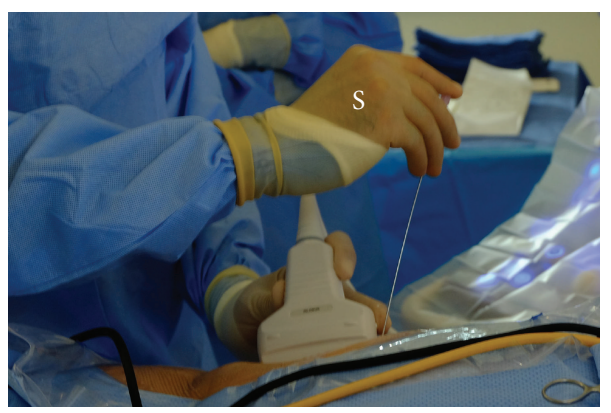

(c)

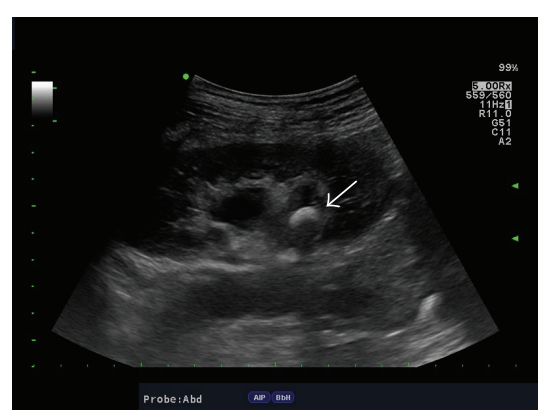

(b)

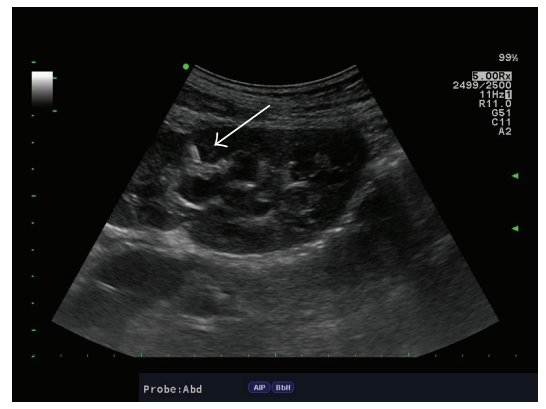

(d)

FIGURE 1: Establishing renal access using ultrasound guidance. (a) The operative surgeon (S) holds the ultrasound probe and the assistant (A) holds a syringe attached to ureteral catheter for normal saline infusion if needed. (b) Ultrasonographic image of the kidney along its longitudinal axis demonstrating the stone in the renal pelvis (white arrow) within a mildly hydronephrotic collecting system. (c) During the needle insertion, the operative surgeon $(S)$ holds both the ultrasound probe and the needle to perform the puncture. For hand positioning, the nondominant hand holds the ultrasound probe while the dominant hand holds the needle. (d) The needle can be visualized (white arrow) entering the collecting system through upper pole calyx in this case.

patient populations, including pregnant [8] and pediatric patients [9]. Others have demonstrated the feasibility and usefulness of complete ultrasound guidance in PCNL using a supine position [10-12].

In this study, we report our experience with ultrasound guidance for renal access and tract dilation in prone PCNL. We also evaluate the feasibility of this technique in the minimally dilated collecting system.

\section{Patients and Methods}

This was a prospective observational cohort study completed at two academic medical centers, University of California, San Francisco (UCSF), and San Francisco General Hospital (SFGH). In May of 2014, the operative surgeon for this study (TC) changed his approach for renal puncture from fluoroscopy guided access to ultrasound-guided access. Prior to that, PCNL had been performed under fluoroscopy guidance alone. After institutional IRB was obtained, clinical data for all patients presenting between March and August 2015 requiring treatment with PCNL was collected. Inclusion criteria for this study were (1) patients with renal or proximal ureteral stone, (2) age greater than 18 years. No patients were excluded from this analysis during the study time frame. Procedures performed during this time period utilized ultrasound guidance for all steps of the renal access and dilation. All procedures were performed by a single surgeon
(TC) and perioperative data was collected prospectively for these analyses.

Preoperatively, complete blood count, serum creatinine, and demographic data were obtained from all patients. Noncontrast CT was used to determine stone characteristics. Ultrasound-guided cases were compared to a control cohort of cases done with fluoroscopic guidance by the same surgeon prior to the adoption of ultrasound guidance and matched for stone size to the ultrasound-guided group. Student's $t$-test and Chi-square test were used to compare differences between the two groups and statistical analyses were performed using Stata/IC version 13.1 (StataCorp, Texas, USA). Data are expressed as mean \pm standard deviation or percentage with a significance level of $p<0.05$.

After induction of general anesthesia, an open-ended 5-French ureteral catheter was inserted into the ipsilateral ureter for approximately 20 centimeters under cystoscopic guidance from a supine position with the patient on the transport gurney. Patients were then moved to a prone position and safely secured to the operative table. Percutaneous renal access was obtained by the operative surgeon using ultrasound guidance without a needle guide. We used a 3.5$\mathrm{MHz}$ convex abdominal transducer (Hitachi Aloka Medical) to localize the stone position as well as an ideally suited posterior calyx for puncture (Figures 1(a) and 1(b)). An 18gauge Echotip needle (Cook Medical) was advanced under real-time ultrasound monitoring (Figures 1(c) and 1(d)). In 


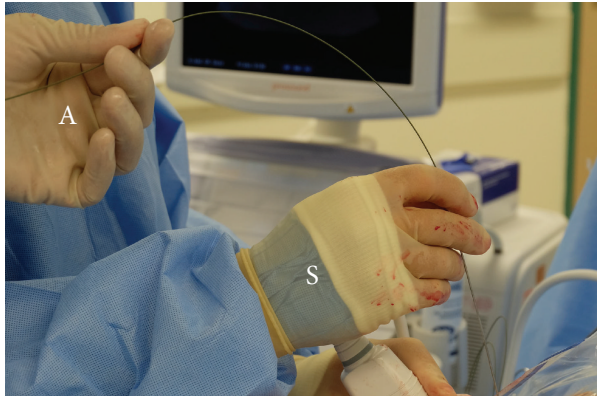

(a)

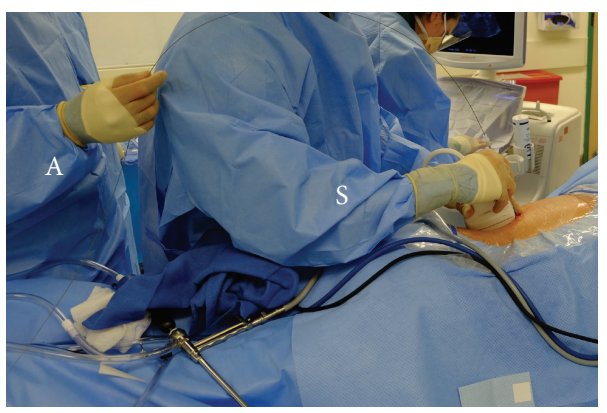

(c)

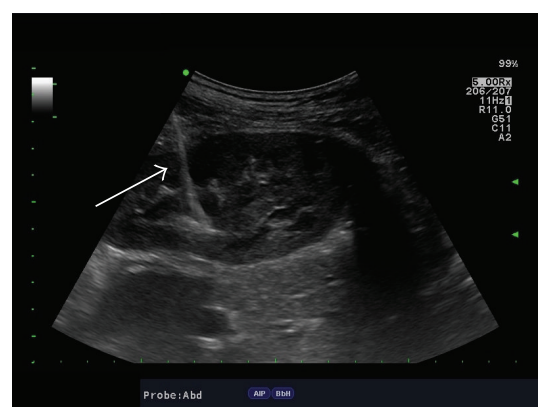

(b)

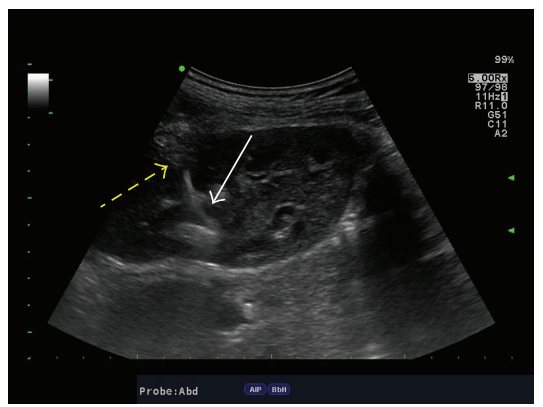

(d)

FIGURE 2: Introduction of working wire and dilators using ultrasound guidance. (a) A J-tip coaxial wire is inserted through the needle after its tip is confirmed in the collecting system. The operative surgeon (S) controls the needle with dominant hand and holds the ultrasound probe with the nondominant hand while the assistant (A) controls the wire. (b) Ultrasonographic image of the kidney demonstrating the wire (white arrow) in the collecting system after needle withdrawal. Its appearance is highly echogenic and prominent. (c) For fascial dilation, a 10-French fascial dilator is passed over the wire. Again, the operative surgeon (S) holds the ultrasound probe and controls the dilator while the assistant (A) controls the wire. (d) As the fascial dilator passes over the working wire, the wire is seen very clearly as an echogenic line (white arrow). The dilator obscures the view of the wire and this interface of the echogenic wire and the area where the wire disappears from view is the tip of the fascial dilator (yellow arrow).

the absence of hydronephrosis, saline was injected retrograde through the ureteral catheter to dilate the collecting system for easier imaging though this was not routinely done for every patient. Entry into the collecting system was confirmed with either aspiration or efflux of urine through the puncture needle or clear visualization of the needle tip within the urinary space or touching the renal stone (Figure 1(d)). After entry into the collecting system was confirmed, a Jtip coaxial guide wire (Bard Medical) was inserted into the renal pelvis or down the proximal ureter, using ultrasound imaging to visualize the wire passing into the collecting system (Figures 2(a) and 2(b)). We then sequentially passed a 10-French fascial dilator and a safety wire introducer over the wire. The tip of each instrument was visualized with ultrasound imaging entering the collecting system to prevent collecting system perforation. The wire appeared with a very bright echogenic signal ultrasonographically, while the dilator and safety wire introducer were not echogenic. Their advancement over the wire could be visualized as they obscured the echogenic appearance of the wire. By watching for disappearance of the wire, the exact position of the tip of each dilator was visualized (Figures 2(c) and 2(d)). Using the safety wire introducer, a second safety wire was advanced into the collecting system and imaged as it passed into the collecting system. The balloon was then imaged passing into the collecting system (Figures 3(a) and 3(b)) over one of the wires and a working tract was dilated with a high-pressure balloon dilator (BARD X-Force, Bard Medical) using ultrasonography. Then, either a $24-$ or 30 -French sheath was advanced over the inflated balloon (Figures 3(c) and 3(d)). After removal of the balloon, nephroscopy was performed with a 20.8- or 27-French rigid offset nephroscope (Richard Wolf Medical). Stone fragmentation was accomplished using an ultrasonic CyberWand lithotripter (Olympus America). Flexible nephroscopy was performed to look for residual fragments and holmium laser lithotripsy via a flexible cystoscope was used if needed for their removal. Stone clearance was confirmed intraoperatively with ultrasound imaging and nephroscopy. At the end of the procedure, a nephrostomy tube was placed in all patients under limited fluoroscopic screening. We routinely used a 10-French Cope loop catheter (Cook Medical) for this purpose.

Intraoperatively, fluoroscopic screening was used only for nephrostomy tube placement, positional confirmation, and readjustment using a mobile multidirectional C-arm fluoroscopy unit with an under table X-ray. Renal access puncture time (defined as the time elapsed from initial renal ultrasonographic imaging to successful placement of the needle into the collecting system), tract dilation time with US guidance (defined as the time elapsed from insertion of 


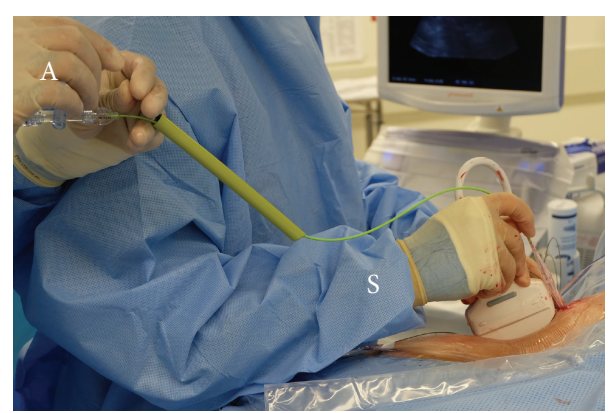

(a)

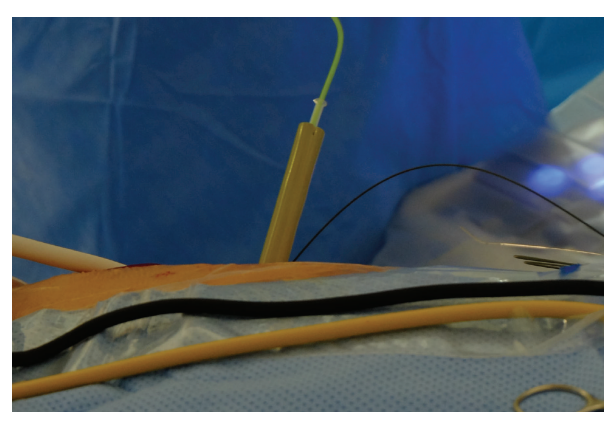

(c)

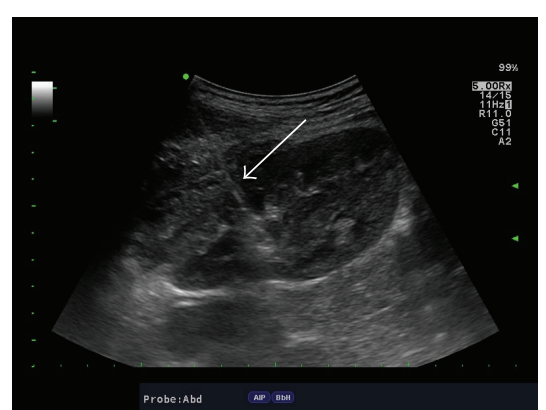

(b)

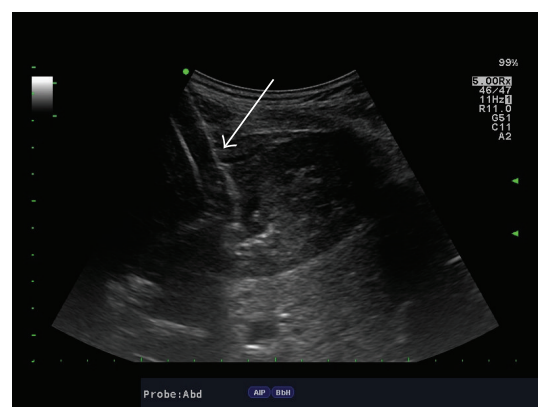

(d)

FIGURE 3: Tract dilation with high-pressure balloon under ultrasound guidance. (a) The deflated balloon dilator is inserted over a working wire and for this step, the operative surgeon (S) controls the ultrasound probe and distal end of the balloon while the assistant (A) controls the wire on the proximal end of the balloon dilator. (b) Ultrasonographic image of the kidney along its longitudinal axis demonstrates that the tip of the deflated balloon dilator (white arrow) is difficult to visualize and differentiate from the wire. (c) The sheath has been inserted over the inflated balloon dilator that is subsequently withdrawn. (d) The inflated balloon (white arrow) can be readily seen with ultrasound imaging.

the wire into the collecting system to advancement of the access sheath over the balloon), fragmentation time (defined as the time elapsed from insertion of the rigid nephroscope to the placement of the nephrostomy tube), estimated blood loss and total operative time (defined as the time elapsed from initial cystoscopy for ureteral catheter placement until the placement of the nephrostomy tube), and postoperative outcomes were also recorded in this study.

\section{Results}

From March to August 2015, thirty-eight patients underwent PCNL where ultrasound was used to guide all steps of renal access and tract dilation and were enrolled in our study. There were 17 males and 21 females with a mean age of $52.7 \pm 17.2$ years and American Society of Anesthesiologists (ASA) physical status classification ranged from class 1 to class 3. Stone size varied from 9.0 to 79.4 millimeters (mean $27.2 \pm 17.6$ millimeters) and only ten of these thirtyeight kidneys demonstrated more than mild hydronephrosis on preoperative imaging (Table 1). Compared to a fluoroscopic control group of 38 cases done by the same surgeon prior to the adoption of ultrasound guidance, both groups were comparable in terms of demographic and preoperative parameters. Body mass index (BMI) was the only difference of note where it was significantly lower in the ultrasound group (26.1 versus $30.3 \mathrm{~kg} / \mathrm{m}^{2}, p=0.03$ ).
Puncture time varied from 9 to 540 seconds with a mean of $135.4 \pm 132.5$ seconds (Table 1). Mean dilation and fragmentation times were $11.5 \pm 3.8$ and $37.5 \pm 29.0$ minutes, respectively. While the upper and lower calices were selected for puncture almost equally in the ultrasound group, the lower calyx was targeted most often in the fluoroscopic group. Mean total operative time was $129.3 \pm 41.1$ minutes, which was not statistically significantly different compared to a control of $147.1 \pm 52.1$ minutes for the fluoroscopic group. While no fluoroscopic screening was required during renal access or tract dilation, fluoroscopic imaging was used for confirmation of nephrostomy tube positioning at the end of the procedure in all cases. For these procedures, mean fluoroscopic screening time was $17.7 \pm 13.3$ seconds (range 1.0 to 63.0 seconds) and mean radiation exposure dose was $3.1 \pm 3.2 \mathrm{mGy}$ (range 0.2 to $14.0 \mathrm{mGy}$ ). Comparatively, in the fluoroscopic control group of patients, total fluoroscopic screening time and radiation exposure were $182.9 \pm 119.0$ seconds and $47.5 \pm 52.3 \mathrm{mGy}$, respectively. Intraoperatively, all patients were visually and ultrasonographically confirmed stone-free, confirmed by KUB and ultrasound performed at 30-day follow-up.

One grade 2 complication as defined by the ClavienDindo classification was encountered during the immediate postoperative period [13]. This was a female patient with a history of recurrent UTI who underwent concurrent contralateral ureteroscopy and laser lithotripsy for a renal stone. 
TABLE 1: Patient characteristics and perioperative parameters.

\begin{tabular}{|c|c|c|c|}
\hline Parameter & $\begin{array}{l}\text { Ultrasound group } \\
\quad(n=38)\end{array}$ & $\begin{array}{c}\text { Fluoroscopy group } \\
(n=38) \\
\end{array}$ & $p$ value \\
\hline \multicolumn{4}{|l|}{ Preoperative characteristics } \\
\hline Mean (SD) age & $52.7 \pm 17.2$ & $52.9 \pm 14.3$ & 0.96 \\
\hline \multicolumn{4}{|l|}{ Gender, $n(\%)$} \\
\hline Male & $17(44.7)$ & $19(50.0)$ & \multirow{2}{*}{0.65} \\
\hline Female & $21(55.3)$ & $19(50.0)$ & \\
\hline Mean (SD) BMI $\left(\mathrm{kg} / \mathrm{m}^{2}\right)$ & $26.1 \pm 7.3$ & $30.3 \pm 8.2$ & 0.03 \\
\hline Mean $(\mathrm{SD})$ preoperative serum creatinine $(\mathrm{mg} / \mathrm{dL})$ & $0.92 \pm 0.33$ & $0.97 \pm 0.37$ & 0.51 \\
\hline Mean (SD) preoperative hematocrit (\%) & $39.0 \pm 5.9$ & $40.1 \pm 4.9$ & 0.38 \\
\hline \multicolumn{4}{|l|}{ ASA physical status, $n(\%)$} \\
\hline Class 1 & $7(18.4)$ & $6(15.8)$ & \multirow{3}{*}{0.36} \\
\hline Class 2 & $17(44.7)$ & $23(60.5)$ & \\
\hline Class 3 & $14(36.9)$ & $9(23.7)$ & \\
\hline \multicolumn{4}{|l|}{ Stone laterality, $n(\%)$} \\
\hline Right & $15(39.5)$ & $16(42.1)$ & \multirow{2}{*}{0.82} \\
\hline Left & $23(60.5)$ & $22(57.9)$ & \\
\hline \multicolumn{4}{|l|}{ Stone type and position, $n(\%)$} \\
\hline Calyceal renal stone & $12(31.6)$ & $9(23.7)$ & \multirow{5}{*}{0.24} \\
\hline Renal pelvic stone & $10(26.3)$ & $14(36.8)$ & \\
\hline Staghorn stone & $6(15.8)$ & $6(15.8)$ & \\
\hline Proximal ureteral stone & $3(7.9)$ & $7(18.4)$ & \\
\hline Multiple stones & $7(18.4)$ & $2(5.3)$ & \\
\hline Mean (SD) stone size (millimeters) & $27.2 \pm 17.6$ & $28.5 \pm 14.5$ & 0.72 \\
\hline \multicolumn{4}{|l|}{ Degree of hydronephrosis, $n(\%)$} \\
\hline None & $20(52.6)$ & $15(39.5)$ & \multirow{4}{*}{0.59} \\
\hline Mild & $8(21.1)$ & $13(34.2)$ & \\
\hline Moderate & $8(21.1)$ & $8(21.1)$ & \\
\hline Severe & $2(5.2)$ & $2(5.2)$ & \\
\hline \multicolumn{4}{|l|}{ Intraoperative measurements } \\
\hline Mean (SD) puncture time (seconds) & $135.4 \pm 132.5$ & NA & \\
\hline \multicolumn{4}{|l|}{ Puncture location, $n(\%)$} \\
\hline Upper calyx & $16(42.1)$ & $3(7.9)$ & \multirow{3}{*}{0.00} \\
\hline Middle calyx & $7(18.4)$ & $1(2.6)$ & \\
\hline Lower calyx & $15(39.5)$ & $34(89.5)$ & \\
\hline Mean (SD) dilation time (minutes) & $11.5 \pm 3.8$ & NA & \\
\hline Mean (SD) fragmentation time (minutes) & $37.5 \pm 29.0$ & NA & \\
\hline Mean (SD) total operative time (minutes) & $129.3 \pm 41.1$ & $147.1 \pm 52.1$ & 0.11 \\
\hline Mean $(\mathrm{SD})$ radiation exposure dose (mGy) & $3.1 \pm 3.2$ & $47.5 \pm 52.3$ & 0.00 \\
\hline Mean (SD) fluoroscopic screening time (seconds) & $17.7 \pm 13.3$ & $182.9 \pm 119.0$ & 0.00 \\
\hline \multicolumn{4}{|l|}{ Postoperative outcomes } \\
\hline Mean $(\mathrm{SD})$ postoperative serum creatinine $(\mathrm{mg} / \mathrm{dL})$ & $0.92 \pm 0.37$ & $1.01 \pm 0.39$ & 0.33 \\
\hline Mean (SD) difference in preoperative and postoperative hematocrit (\%) & $4.0 \pm 5.6$ & $2.6 \pm 3.5$ & 0.18 \\
\hline Mean (SD) hospital stay (days) & $3.1 \pm 1.4$ & $2.9 \pm 1.9$ & 0.67 \\
\hline \multicolumn{4}{|l|}{ Postoperative complication, $n(\%)$} \\
\hline Grade 1 & 0 & 0 & \multirow{3}{*}{0.56} \\
\hline Grade 2 & $1(2.6)$ & 0 & \\
\hline Grade 3 & 0 & $2(5.3)$ & \\
\hline \multicolumn{4}{|l|}{ Stone-free status, $n(\%)$} \\
\hline Stone-free & $38(100)$ & $34(89.4)$ & \multirow{3}{*}{0.12} \\
\hline Insignificant residual stone & 0 & $2(5.3)$ & \\
\hline Significant residual stone & 0 & $2(5.3)$ & \\
\hline
\end{tabular}


She experienced prolonged fever postoperatively and was managed successfully with broad-spectrum antibiotics.

On the morning of the first postoperative day, creatinine was not statistically significantly changed compared to preoperative values. Change in hematocrit was not statistically significantly different between the ultrasound and fluoroscopic groups and no blood transfusions were necessary in either group. No additional complications were seen at follow-up of 30 days. No secondary procedures were required for all patients (Table 1).

\section{Discussion}

Percutaneous nephrolithotomy is the primary procedure used for the management of patients with large renal stones not amenable to ureteroscopy or shockwave lithotripsy. In the United States and around the world, fluoroscopic guidance has been the primary imaging method of choice used to guide percutaneous renal access and establish the working tract to facilitate these procedures. Over time, concerns have grown that long-term cumulative ionizing radiation exposure may possibly increase the incidence of malignancies [14-16]. For nephrolithiasis patients, reducing their exposure to ionizing radiation in all settings is an important goal as these patients are at high risk for increased cumulative radiation exposure compared to patient populations without nephrolithiasis [17]. Intraoperative radiation exposure to surgical staff not only results from direct exposure to the fluoroscopic radiation beam that is in close proximity to many staff members, it also stems from beam scatter produced from the interaction between the primary radiation beam with the patient and the operating table $[4,6]$. While some have shown that during PCNL radiation exposure may be relatively low, practices may vary from place to place and case to case [5]. Despite usage of protective equipment, of all the operating room personnel, the surgeon generally receives the maximum ionizing radiation exposure, mostly to the legs and the eyes [6].

Several methods have been proposed to facilitate renal access while reducing radiation exposure for PCNL. One such alternative is endoscopic guidance, commonly known as Endoscopic Combined IntraRenal Surgery (ECIRS). This technique begins with retrograde access into the renal collecting system using a flexible ureteroscope. After positioning the ureteroscope in the target calyx of choice, fluoroscopy is used to guide a needle into the kidney in an antegrade percutaneous fashion and the needle enters the collecting system under direct vision of the ureteroscope. It can be performed either in a modified supine [18] or prone splitleg position $[19,20]$. Once a target calyx is identified by flexible ureterorenoscopy, fluoroscopy is still needed for caliceal puncture. Compared to standard PCNL, however, this technique can be associated with lower fluoroscopic screening time and increased stone clearance $[19,21]$.

Real-time diagnostic ultrasonography (US) is becoming more widely accepted as an alternative imaging modality for directing PCNL in a dilated renal collecting system. The overall success rate can be comparable to that of standard fluoroscopic-guided PCNL [22]. Ultrasonography is free of ionizing radiation and effective and offers an advantage of portability compared to fluoroscopy. In addition, it provides additional imaging information over fluoroscopy during PCNL, including imaging and identification of viscera and structures that might be located between the skin and kidney, the depth of penetration of the puncture needle relative to the target calyx, and an easier means of differentiating posterior from anterior calyces. For these reasons, ultrasonography can help prevent adjacent and visceral organ injury such as inadvertent colonic penetration. In addition, there is no need for routine retrograde contrast or fluid injection and therefore the use of ultrasonography eliminates the need for a retrograde ureteral catheter if the surgeon is faced with the problem of unsuccessful retrograde ureteral catheterization [23]. Given its radiation-free nature, it is also an ideal imaging guidance modality for patient populations more sensitive to radiation exposure, including pediatric and pregnant patients [24]. Moreover, the use of US at the end of the procedure helps the urologist to look for nonopaque and semiopaque residual stones that are not visualized by radiography to confirm stone-free status intraoperatively [25].

In an attempt to minimize ionizing radiation exposure during PCNL, renal access with ultrasound guidance was first reported by Desai et al. in 1999. PCNL was done with US-guided percutaneous puncture in 45 renal units for 40 pediatric patients. However, fluoroscopic screening was still required during tract dilation. Overall stone clearance rate of $91 \%$ was achieved with few minor complications [26]. The largest published series of ultrasound-guided PCNL comes from Li et al. In 2014, they reported 8025 cases performed by a single surgeon [27] demonstrating a final stone-free rate of $85.5 \%$. Yan et al. demonstrated a similar stone-free rate with their series of 679 patients [25]. Our series differs from all previously mentioned studies in that we demonstrate that ultrasound imaging can be used to reliably visualize and guide all steps of renal access and tract dilation without having to rely on fluoroscopic screening, supine positioning, or a two-step direct visualization method.

This is also, to our knowledge, the first series to describe that this technique and approach can be used to successfully gain renal access for PCNL in any degree of hydronephrosis. In our experience, while placement of a ureteral catheter and subsequent infusion of a small amount of normal saline could help identify calyces for puncture, we did not find that large volume injection was necessary. We were successful in gaining renal access even in the absence of moderate or severe hydronephrosis and all of our procedures were performed with the patient in the prone position. With modern ultrasound imaging consoles, the nondilated system can be visualized well even in the prone position, and it may be that improvement in imaging technology and quality since Desai's first publication has made an ultrasound-guided approach to PCNL more feasible and less risky for patients.

Using ultrasound to guide PCNL is not without its technical challenges, however. In our practice, a high-pressure balloon dilator is routinely used for tract establishment. One difficulty with accurate placement of the balloon with ultrasonography is that the deflated balloon tip is not easily visualized under ultrasound guidance. Once inflated, the 
balloon can readily be seen, but its tip can be difficult to accurately identify in the deflated state. We overcame this problem by constantly moving the wire back and forth while passing the balloon over it and looking for a change in the wire contour to judge where the tip of the balloon was relative to the wire. Despite this, the tip of the balloon could still be difficult to distinctly visualize. In addition, since ultrasound imaging relies on real-time movement of the probe over the patient's body and the probe is not fixed in place, one technical challenge presented is the need to keep the ultrasound probe on the operative field while passing the wire and dilation instruments into the kidney. With the technology currently available, these multiple tasks require at minimum two sets of hands in order to be performed efficiently. As demonstrated in Figures 1-3, we standardized our procedures to minimize the amount of assistance required by the primary surgeon by having one assistant stand behind the surgeon toward the foot of the bed. In this fashion the operative surgeon performed all steps of renal access and tract dilation while holding the ultrasound probe with the nondominant hand in place over the kidney while the assistant held control of the wire to facilitate dilation. Accomplishing this, however, requires a coordinated team effort. Lastly, with ultrasound imaging, the needle, wire, and dilators can readily be imaged but the nephrostomy tube can often be difficult to visualize, highlighting an area of possible future technology development For this reason, we used limited fluoroscopic imaging to confirm adequate placement of the nephrostomy tube at the end of each case, as it was difficult to perform accurately with ultrasound imaging guidance alone.

Two significant differences between our cohorts that warrant discussion were a lower BMI in the ultrasound group and a higher use of the lower pole in the fluoroscopic group. These differences may have influenced our results. For example, our clinical outcomes, including stone-free and complication rates may have been higher in the ultrasound group had they been comprised of patients with a higher BMI. On the other hand, the higher rate of upper pole entry in the ultrasound group may have increased the likelihood of a complication such as pneumothorax in this group, though these differences were not seen. In balance, we appeared to perform both techniques with similar associated complication rates despite these differences. These results may therefore be more likely reflective of surgeon ability as opposed to patient characteristics. The best way to know this with greater certainty would be to perform a randomized trial for surgical technique, which was not the aim of this current study. While our ultrasound cohort is relatively small and consists of patients with a relatively low BMI, we present this series as a prospective, descriptive study in the hopes that the practicing urologist will be encouraged to utilize intraoperative ultrasonography for all steps of renal tract access and dilation during PCNL. In our series, the operative surgeon transitioned from fluoroscopic to ultrasound image guidance by performing a limited number of mentored cases over a short period of time. In this short period of time, applying ultrasound guidance to all steps of renal access and dilation was associated with a significant reduction in radiation exposure for intraoperative personnel and the patient.
Particularly for the urologist who relies on fluoroscopy to guide percutaneous renal stone surgery, the techniques described with our experience may have significant relevance.

\section{Conclusions}

Ultrasound-guided technique for renal access and tract dilation in prone percutaneous nephrolithotomy is feasible and effective in treating renal stones. It can be performed successfully and safely even in the patients with a minimally dilated collecting system in the prone position. Our experience demonstrates good surgical outcomes with significant reduction in fluoroscopic screening time to the patient, surgeon, and intraoperative personnel.

\section{Conflict of Interests}

The authors declare that there is no conflict of interests regarding the publication of this paper.

\section{Authors' Contribution}

Thomas Chi, Selma Masic, and Manint Usawachintachit contributed to data collection and analysis as well as paper drafting. Thomas Chi and Jianxing Li contributed to study design, paper revision, and supervision of this study.

\section{Acknowledgment}

This study is supported by Multidisciplinary K12 Urologic Research Career Development Program (TC), K12-DK-07006.

\section{References}

[1] S. R. Patel and S. Y. Nakada, "The modern history and evolution of percutaneous nephrolithotomy," Journal of Endourology, vol. 29, no. 2, pp. 153-157, 2015.

[2] N. Hidajat, P. Wust, M. Kreuschner, R. Felix, and R.-J. Schröder, "Radiation risks for the radiologist performing transjugular intrahepatic portosystemic shunt (TIPS)," British Journal of Radiology, vol. 79, no. 942, pp. 483-486, 2006.

[3] E. Vano, N. J. Kleiman, A. Duran, M. Romano-Miller, and M. M. Rehani, "Radiation-associated lens opacities in catheterization personnel: results of a survey and direct assessments," Journal of Vascular and Interventional Radiology, vol. 24, no. 2, pp. 197204, 2013.

[4] G. O. Hellawell, S. J. Mutch, G. Thevendran, E. Wells, and R. J. Morgan, "Radiation exposure and the urologist: what are the risks?” The Journal of Urology, vol. 174, no. 3, pp. 948-952, 2005.

[5] G. Kumari, P. Kumar, P. Wadhwa, M. Aron, N. P. Gupta, and P. N. Dogra, "Radiation exposure to the patient and operating room personnel during percutaneous nephrolithotomy," International Urology and Nephrology, vol. 38, no. 2, pp. 207-210, 2006.

[6] H. S. Majidpour, "Risk of radiation exposure during PCNL," Urology Journal, vol. 7, no. 2, pp. 87-89, 2010. 
[7] A. Tepeler, M. Binbay, E. Yuruk et al., "Factors affecting the fluoroscopic screening time during percutaneous nephrolithotomy," Journal of Endourology, vol. 23, no. 11, pp. 1825-1829, 2009.

[8] A. Fregonesi, F. G. F. Dias, R. D. Saade, V. Dechaalani, and L. O. Reis, "Challenges on percutaneous nephrolithotomy in pregnancy: supine position approach through ultrasound guidance," Urology Annals, vol. 5, no. 3, pp. 197-199, 2013.

[9] F. Sharifiaghdas, A. Tabibi, A. Nouralizadeh et al., "Our experience with totally ultrasonography-guided percutaneous nephrolithotomy in children," Journal of Endourology, 2015.

[10] M. Etemadian, M. Amjadi, and N. Simforoosh, "Transcutaneous ultrasound guided nephrolithotomy: the first report from Iran," The Journal of Urology, vol. 1, no. 2, pp. 82-84, 2004.

[11] W. M. Gamal, M. Hussein, M. Aldahshoury et al., "Solo ultrasonography-guided percutanous nephrolithotomy for single stone pelvis," Journal of Endourology, vol. 25, no. 4, pp. 593596, 2011.

[12] M. M. Hosseini, A. Hassanpour, R. Farzan, A. Yousefi, and M. A. Afrasiabi, "Ultrasonography-guided percutaneous nephrolithotomy," Journal of Endourology, vol. 23, no. 4, pp. 603-607, 2009.

[13] J. J. de la Rosette, D. Opondo, F. P. Daels et al., "Categorisation of complications and validation of the Clavien score for percutaneous nephrolithotomy," European Urology, vol. 62, no. 2, pp. 246-255, 2012.

[14] K. Faulkner and E. Vañó, "Deterministic effects in interventional radiology," Radiation Protection Dosimetry, vol. 94, no. 1-2, pp. 95-98, 2001.

[15] T. B. Wenzl, "Increased brain cancer risk in physicians with high radiation exposure," Radiology, vol. 235, no. 2, pp. 709-711, 2005.

[16] M. S. Linet, D. M. Freedman, A. K. Mohan et al., "Incidence of haematopoietic malignancies in US radiologic technologists," Occupational and Environmental Medicine, vol. 62, no. 12, pp. 861-867, 2005.

[17] A. C. Westphalen, R. Y. Hsia, J. H. Maselli, R. Wang, and R. Gonzales, "Radiological imaging of patients with suspected urinary tract stones: national trends, diagnoses, and predictors," Academic Emergency Medicine, vol. 18, no. 7, pp. 699-707, 2011.

[18] C. M. Cracco and C. M. Scoffone, "ECIRS (Endoscopic Combined IntraRenal Surgery) in the Galdakao-modified supine Valdivia position: a new life for percutaneous surgery?" World Journal of Urology, vol. 29, no. 6, pp. 821-827, 2011.

[19] A. G. Lantz, P. O’Malley, M. Ordon, and J. Y. Lee, “Assessing radiation exposure during endoscopic-guided percutaneous nephrolithotomy," Journal of the Canadian Urological Association, vol. 8, no. 9-10, pp. 347-351, 2014.

[20] S. Hamamoto, T. Yasui, A. Okada et al., "Endoscopic combined intrarenal surgery for large calculi: simultaneous use of flexible ureteroscopy and mini-percutaneous nephrolithotomy overcomes the disadvantageous of percutaneous nephrolithotomy monotherapy," Journal of Endourology, vol. 28, no. 1, pp. 28-33, 2014.

[21] W. Isac, E. Rizkala, X. Liu, M. Noble, and M. Monga, "Endoscopic-guided versus fluoroscopic-guided renal access for percutaneous nephrolithotomy: a comparative analysis," Urology, vol. 81, no. 2, pp. 251-256, 2013.

[22] S. Andonian, C. M. Scoffone, M. K. Louie et al., "Does imaging modality used for percutaneous renal access make a difference? A matched case analysis," Journal of Endourology, vol. 27, no. 1, pp. 24-28, 2013.
[23] B. Lojanapiwat, "The ideal puncture approach for PCNL: fluoroscopy, ultrasound or endoscopy?" Indian Journal of Urology, vol. 29, no. 3, pp. 208-213, 2013.

[24] N. Penbegul, H. Soylemez, Y. Bozkurt et al., "An alternative and inexpensive percutaneous access needle in pediatric patients," Urology, vol. 80, no. 4, pp. 938-940, 2012.

[25] S. Yan, F. Xiang, and S. Yongsheng, "Percutaneous nephrolithotomy guided solely by ultrasonography: a 5-year study of $>700$ cases," BJU International, vol. 112, no. 7, pp. 965-971, 2013.

[26] M. Desai, V. Ridhorkar, S. Patel, S. Bapat, and M. Desai, "Pediatric percutaneous nephrolithotomy: assessing impact of technical innovations on safety and efficacy," Journal of Endourology, vol. 13, no. 5, pp. 359-364, 1999.

[27] J. Li, B. Xiao, W. Hu et al., "Complication and safety of ultrasound guided percutaneous nephrolithotomy in 8, 025 cases in China," Chinese Medical Journal, vol. 127, no. 24, pp. 4184-4189, 2014. 


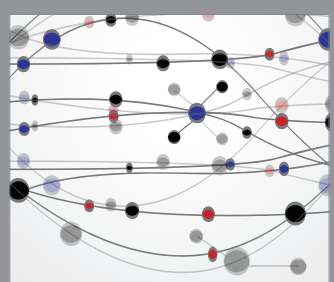

The Scientific World Journal
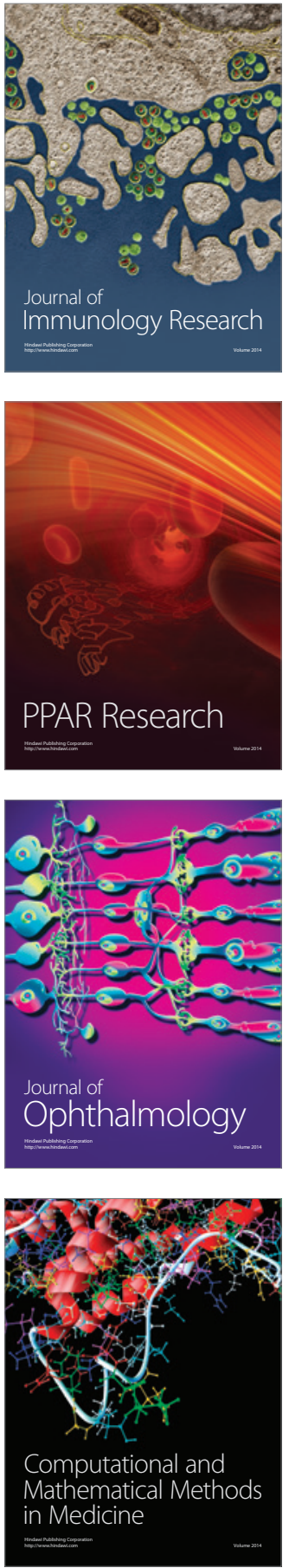

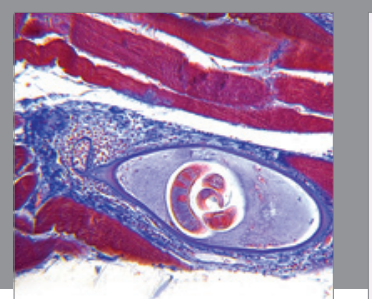

Gastroenterology Research and Practice

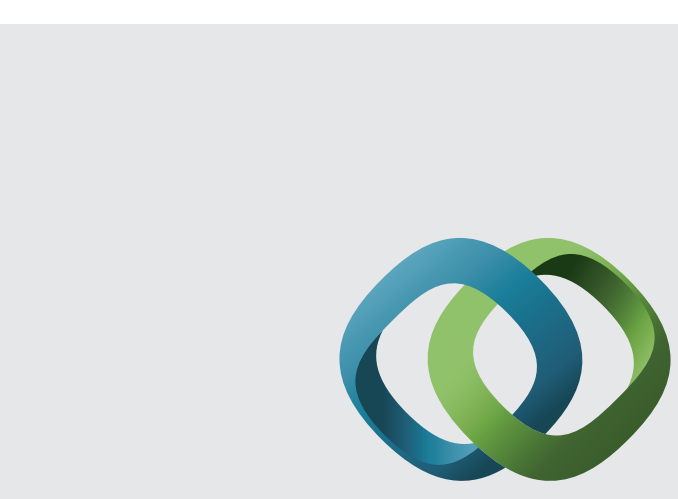

\section{Hindawi}

Submit your manuscripts at

http://www.hindawi.com
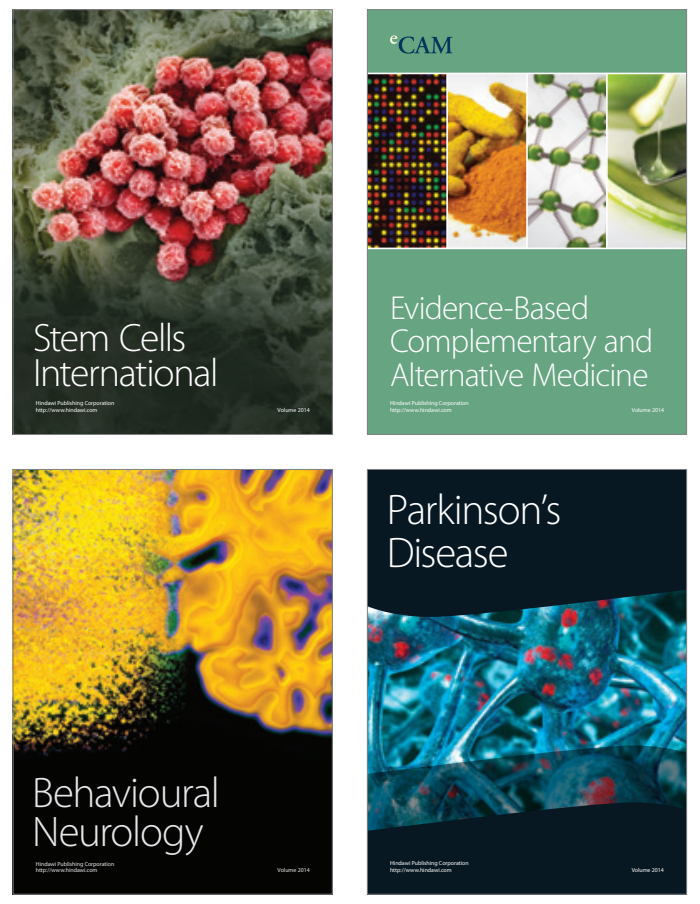
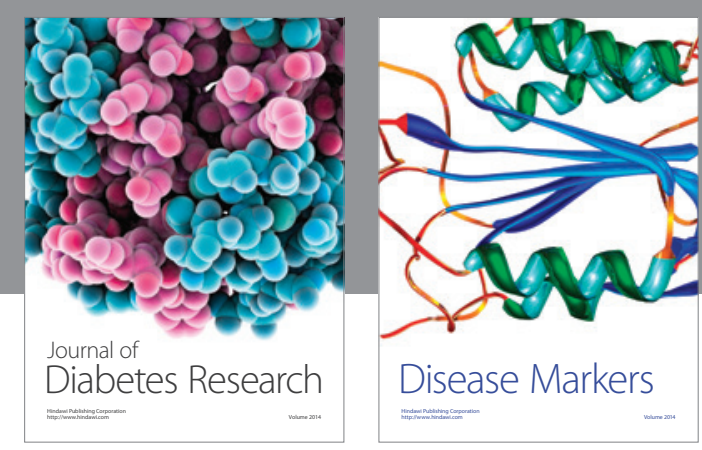

Disease Markers
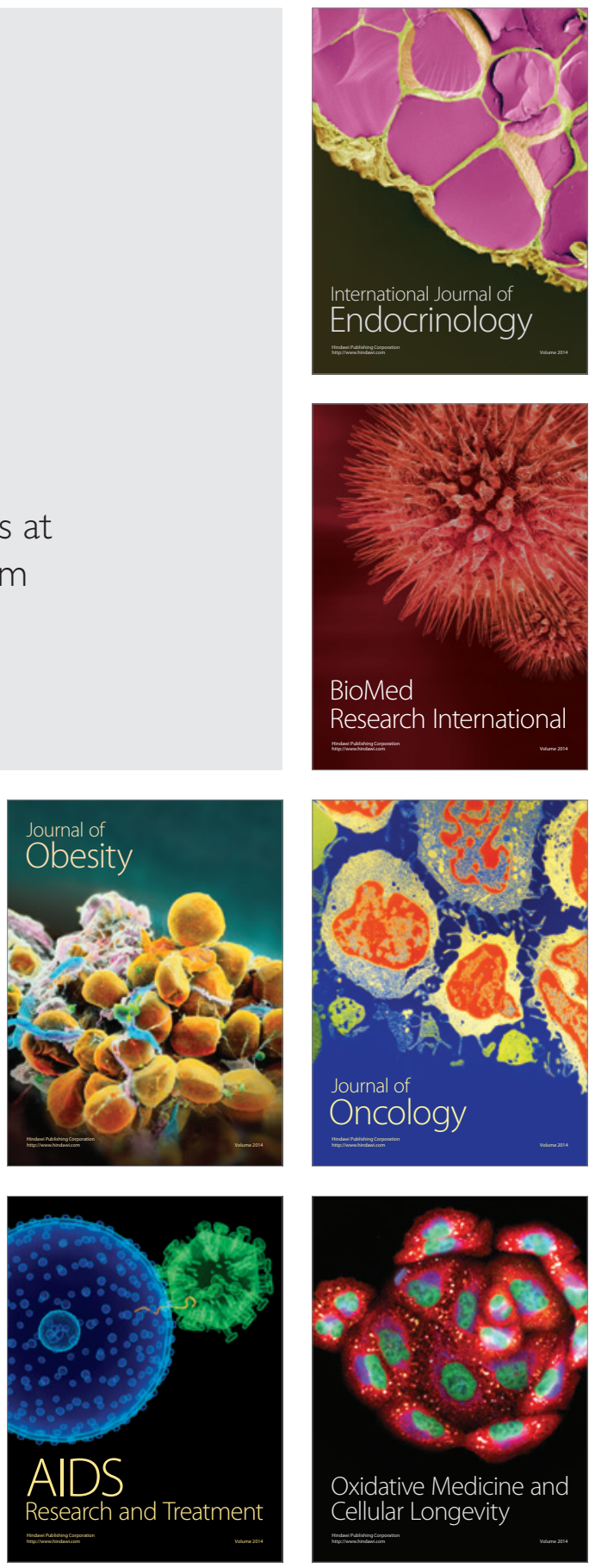